\title{
On Inferring the Refractive-Index Structure of the Tropo- sphere From Electromagnetic Scattering Experiments ${ }^{1}$
}

\author{
Paul L. Smith, Jr. \\ Midwest Research Institute, Kansas City, Mo. \\ (Received November 17, 1964, revised January 21, 1965)
}

\begin{abstract}
Determining the refractive-index structure associated with the scattering of electromagnetic waves by the troposphere is a problem of considerable interest. This paper emphasizes the fact that the refractive-index profile cannot be inferred from scattering measurements at only one wavelength and one scattering angle. Such measurements determine only the spatial Fourier spectrum of the refractive index, at a particular wave number determined by the wavelength and the scattering angle. This point is illustrated by a set of hypothetical refractive-index profiles that cannot be distinguished by measurements at one wavelength and one scattering angle. Methods of obtaining further information about the atmospheric refractive-index structure are reviewed briefly. The suggestion is made that measuring the cross sections of radar angels as a function of the pulse length would provide useful information.
\end{abstract}

\section{Introduction}

The occurrence of electromagnetic scattering from regions of the troposphere that appear visually to be devoid of scattering objects is well established. Examples of this phenomenon include over-the-horizon propagation by tropospheric scattering [IRE, 1955] and the anomalous radar echoes known as "angels" [Battan, 1959]. The origin of the scattered signal in such cases is believed to be related to the variations of the atmospheric refractive index in the scattering volume. Consequently, there is much interest in determining the specific refractive-index structure associated with the observed scattering and to improve the design of tropospheric-scatter communication systems as well as to increase knowledge of the microstructure of the atmosphere.

The purpose of this paper is to designate the kind of information about the refractive-index structure of the troposphere that can be inferred from observations of electromagnetic scattering. In section 2 , it will be shown that measurement of the scattered intensity at a given electromagnetic wavelength and scattering angle does not provide sufficient information to determine the refractive-index profile. Subsequent sections will discuss additional means of investigation that can be used to obtain a more comprehensive description of the atmospheric refractive-index structure.

${ }^{1}$ This paper was presented at the World Conference on Radio Meteorology, Sept. 14-18, 1964, National Bureau of Standards, Boulder, Colo.

\section{Relation Between the Scattered Intensity and the Refractive-Index Profile}

In early analytical work on radio-wave scattering by the troposphere [Friend, 1949], various forms were assumed for the spatial variation of refractive index. Reflection coefficients were then determined from the solutions to the wave equation for the assumed refractive-index profile. A different procedure was followed by Booker and Gordon [1950], who expressed the refractive-index structure in terms of the spatial correlation function of the refractive-index fluctuations. For present purposes, however, the refractive index will be represented in terms of its spatial Fourier spectrum, following Megaw [1950]. Thus, let $\Phi(k)$ be the Fourier "power" spectrum of the refractiveindex variations $n(x)$ along the direction bisecting the angle between the incident and scattered directions, as shown in figure 1 . Then the scattered intensity $I_{s}$ is proportional to the value of $\Phi$ at a wave number $k$ determined by the wavelength of the incident signal and the scattering angle. Specifically [Wheelon, 1959; Tatarski, 1961],

$$
I_{s} \alpha \Phi[4 \pi \sin (\theta / 2) / \lambda]
$$

where $\theta$ is the scattering angle, defined in the usual way, and $\lambda$ is the wavelength of the incident electromagnetic signal. 


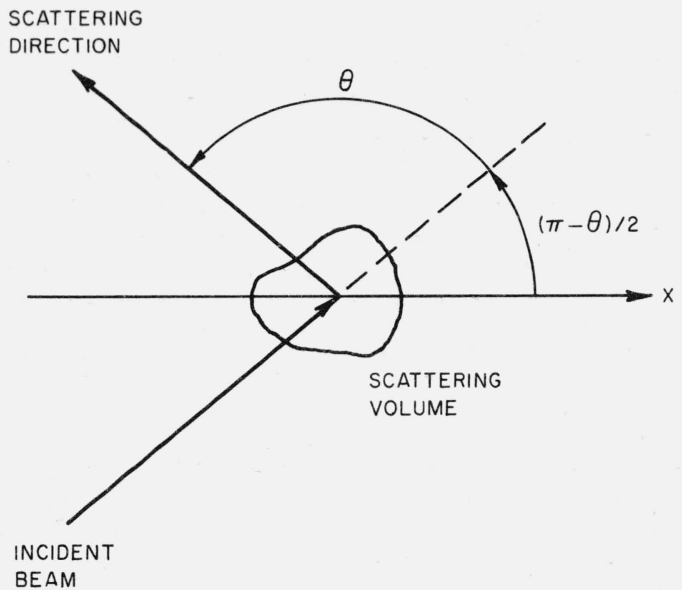

FIGURE 1. Configuration of a scattering experiment.

For the particular case of monostatic radar, where the backscattered intensity is measured, $\theta=180^{\circ}$ and

$$
I_{s} \alpha \Phi[4 \pi / \lambda] \text {. }
$$

This case pertains to observations of radar angels; the "power" spectrum is that measured along the line-of-sight of the radar. The scattered intensity is proportional to the value of $\Phi$ at the wave number $k=4 \pi / \lambda$ corresponding to a wavelength equal to onehalf the radar wavelength.

On the basis of (1), the occurrence of electromagnetic scattering from a region of the troposphere that appears visually to be devoid of scatterers indicates that the value of $\Phi$ at the wave number $k=4 \pi \sin (\theta / 2) / \lambda$ is appreciable in that region. However, the measurement of $\Phi$ at a particular wave number does not give sufficient information to deduce the refractive-index structure of the atmosphere. From measurements at a single wave number, one cannot distinguish among steep gradients, Benard cells, turbulent regions, or other models that may be suggested to account for the observed scattering. There are an infinite number of possible refractive-index profiles that are all equally capable of accounting for the observed scattered intensity. Consequentiy, additional information will be necessary if a satisfactory description of the refractive-index profile is to be obtained.

The four hypothetical one-dimensional refractiveindex profiles shown in figure 2 illustrate the points made above. Accompanying each profile is the corresponding Fourier "power" spectrum. Measurements at a single wave number (such as $k_{1}$ in fig. 2) could not determine which of these four profiles (or the infinity of additional possibilities) actually represents the atmospheric refractive-index profile.

There are several points of interest in connection with the profiles depicted in figure 2. Profile (a) represents a single sharp discontinuity in the refractive
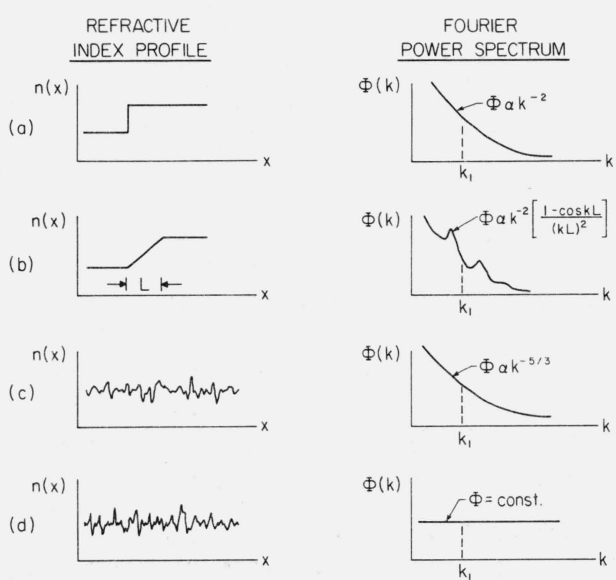

FIGURE 2. Four hypothetical profiles of refractive index and their associated Fourier spectra.

(a) Step discontinuity of refractive index.

(b) Steep gradient of refractive index.

(c) Region of turbulent mixing (Tatarski model).

(d) Completely random variation of refractive index.

index. Of course such a discontinuity is not likely to exist in the atmosphere. However, a change in the refractive index occurring over a distance small compared to the wavelength can be described approximately by such a discontinuity. In any case, the step profile is often a useful model for estimating the orderof-magnitude of variations in refractive index required to account for the observed scattering.

Profile (b) represents a steep gradient of refractive index. Such a profile has been suggested to account for observed radar angels [Hay and Reid, 1962]. When the spatial extent $L$ of the gradient is comparable to $\lambda / 4$, the "power" spectrum $\Phi(4 \pi / \lambda)$ of this profile has a large value. Thus, if strong scattering is to occur, the extent of any such steep gradient must be of the order of one-quarter of the wavelength.

Profile (c) depicts a region of turbulent mixing having the inverse power-law spectrum derived by Tatarski [1961] from theoretical considerations. His arguments for the existence of this type of profile are persuasive; however, Bolgiano's [1963] forwardscatter observations are not in universal agreement with the form of the spectrum as derived by Tatarski.

Profile (d) represents a refractive-index pronile comprising "white noise," i.e., a completely random variation of refractive index with distance. There is no reason to believe such a profile would ever exist in the atmosphere. However, the point of the present argument is that, considering only observations of scattering at a single wavelength and scattering angle, it is not possible to reject such a profile in favor of any of the others.

These one-dimensional profiles give an oversimplified picture of the refractive-index structure of the atmosphere. Actually, the refractive index is a function of all three space coordinates, and the scattering properties of a volume of the atmosphere depend on its three-dimensional power spectrum. Nevertheless, 
the one-dimensional profiles illustrate the fact that only a small amount of information is obtainable from measurements at a single wavelength and scattering angle; the same limitations hold in the three-dimensional case.

\section{Methods of Obtaining Additional Infor- mation About the Refractive-Index Profile}

Observations of electromagnetic scattering at a single wavelength and scattering angle have been shown to be insufficient to define the refractive-index profile in the volume of the troposphere from which the scattering arises. Consequently, additional information must be obtained if further knowledge of the refractive-index structure is desired. There are four possible avenues of approach to the further quantitative study of the atmospheric refractive index: (1) development of theoretical or semiempirical models of the refractive-index structure, (2) direct investigation of this structure using refractometers or similar instruments and the indirect investigation of the refractive index by scattering experiments using (3) additional wavelengths or (4) other scattering angles. A recent paper by Saxton et al., [1964] has discussed these various approaches; only a brief commentary on each approach will be given here.

\subsection{Development of Theoretical Models}

The development of a satisfactory theoretical model of the refractive-index structure of the troposphere would establish the general form of the "power" spectrum $\Phi(k)$; the numerical values of $\Phi$ under the existing conditions could then be determined by measurements at one wave number. This approach is exemplified by the mixing-in-gradient theory developed by Tatarski [1961], by the Benard-cell model, and by the various layer models that have been proposed. The experimental evidence thus far available indicates that no one of the models that have been devised is universally applicable.

\subsection{Direct Investigation by Refractometers}

The refractive-index structure of the troposphere can be studied directly by means of refractometers. A number of suitable refractometers have been developed; among the more successful instruments are the Hay refractometer [Hay et al., 1961] and the Vetter refractometer [Thompson and Vetter, 1958] developed at the National Bureau of Standards. These devices are quite useful for many purposes, but their value in the study of microwave scattering is limited. For example, radar angels are associated with refractiveindex variations having a spatial wavelength equal to one-half the radar wavelength. Consequently, the spatial resolution of the refractometer must also be one-half the radar wavelength or less if the region of the "power" spectrum associated with angels is to be studied.
The resolution of present refractometers falls considerably short of this goal; the problems of refractometer design are such that a resolution of a few centimeters will be extremely difficult to attain. Perhaps a judicious combination of refractometer measurements, down to the resolution limit of the instrument, with extrapolation to shorter wavelengths (higher wave numbers), based on a theoretical model of the refractive-index spectrum, would yield useful results. Such an approach was suggested by Bolgiano [1958], but to the author's knowledge it has not yet been tried.

\subsection{Indirect Investigation Using Additional Wavelengths}

By making scattering measurements at additional wavelengths, the "power" spectrum $\Phi(k)$ can be measured at additional wave numbers. This technique has been adopted by Bolgiano [1963], who employs a bistatic radar system. He uses three wavelengths, and therefore obtains measurements of the Fourier spectrum of the refractive-index profile at three wave numbers. In addition, by means of a beam-swinging technique, he is able to investigate the anisotropy of the refractive-index structure of the troposphere. Bolgiano's experiments have shown that the Tatarski theory of the atmospheric refractive-index structure does not agree consistently with the observed wavelength dependence of the scattering cross section. In fact, the variability exhibited by Bolgiano's data indicates that any effort to devise an accurate theoretical model of the atmospheric refractive-index structure will be faced with formidable difficulties. The multiwavelength method of investigation is very appealing in principle. However, the number of wavelengths needed to obtain an adequate knowledge of the Fourier spectrum of the refractive index is so large as to be prohibitive for practical purposes.

\subsection{Indirect Investigation Using Other Scattering Angles}

Since the wave number $k$ governing the scattering properties of the troposphere depends on the scattering angle, $\Phi(k)$ can also be measured at additional wave numbers by varying the scattering angle. This approach is convenient in laboratory studies of optical scattering by large molecules in solution, and the like. However, the experimental difficulties in studying the scattering properties of the atmosphere as a function of scattering angle would be formidable.

\section{Suggested Radar Backscattering Experi- ment Using a Single Wavelength}

The refractive-index profiles represented in figure 2 are of two different types. Profiles (a) and (b) represent refractive-index variations that could be described as "local," since their spatial extent (in the x-direction, at least) is of the order of the wavelength or less. On the other hand, profiles (c) and (d) represent more ex- 
tensive spatial variations of the refractive index. Profiles of the "local" type have received the most attention in recent discussions of radar angels [Hay and Reid, 1962]. However, the available evidence does not eliminate the possibility of the existence of "extensive" profiles similar to (c) or (d) in figure 2.

Evidence favoring one or the other type of profile ("local" or "extensive") can be obtained by measuring the radar cross sections of angels as a function of the radar pulse length. The cross section arising from a profile of the "local" type (whose spatial extent is of the order of the radar wavelength) would be independent of the pulse length. In contrast, the cross section arising from an "extensive" type of profile would decrease if the pulse length is decreased, because the volume contributing to the echo decreases with pulse length. Therefore, a study of the cross sections of angels as a function of the radar pulse length should distinguish between the two types of refractive-index profile. Experimental evidence that clearly establishes the existence of one or the other type of profile would be very useful in future investigations of electromagnetic scattering by the troposphere.

The practical difficulties that would be encountered in making measurements at various pulse lengths are considerable. The receiver bandwidth would have to be varied to correspond to the spectrum of the transmitted pulse. The minimum detectable signal would increase with the bandwidth, because of increased receiver noise. It would be difficult to make sufficiently accurate measurements of comparative echo intensity over a range of pulse lengths. Nevertheless, in considering experimental studies of the scattering properties of the atmosphere, the possibility of such measurements should not be overlooked.

\section{Conclusions}

(1) Observations of electromagnetic scattering at a single wavelength and scattering angle provide limited information about the refractive-index structure of the troposphere. In particular, it is impossible to infer the refractive-index profile from such observations.

(2) Additional information can be obtained by means of theoretical studies, direct experimental investigation with refractometers, or indirect scattering experiments using additional wavelengths or other scattering angles. Each of these approaches has limitations, and neither separately nor taken together do they provide at present the ability to describe completely the refractive-index structure of the troposphere.
(3) Studies of the radar cross sections of radar angels as a function of the pulse length would also provide useful information about the nature of the refractiveindex structure.

Future advances in understanding the mechanism of electromagnetic scattering by the troposphere will probably come from continued efforts along all these avenues of approach.

This paper is a revised version of an earlier paper [Smith, 1964] that was prepared while the author was a visiting Research Associate at McGill University. The advice and encouragement given by R. R. Rogers and J. S. Marshall have been most helpful. The support provided by the National Science Foundation during the author's tenure at McGill University is also gratefully acknowledged.

\section{References}

Battan, L. J. (1959), Radar meteorology, pp. 133-140 (University of Chicago Press, Chicago, Ill.).

Bolgiano, R., Jr. (1958), The role of turbulent mixing in scatter propagation, IRE Trans. Antennas and Prop. AP-6, 161-168.

Bolgiano, R., Jr. (1963), Evidence of anisotropy in tropospheric microstructure, J. Geophys. Res. 68, No. 16, 4873-4874.

Booker, H. G., and W. E. Gordon (1950), A theory of radio scattering in the troposphere, Proc. IRE 38, No. 4, 401-412.

Friend, A. W. (1949), Theory and practice of tropospheric sounding by radar, Proc. IRE 37, No. 2, 116-138.

Hay, D. R., H. C. Martin, and H. E. Turner (1961), Light-weight refractometer, Rev. Sci. Instr. 32, No. 6, 693-697.

Hay, D. R., and W. M. Reid (1962), Radar angels in the lower troposphere, Can. J. Phys. 40, No. 1, 128-138.

IRE (1955), Scatter propagation issue, Proc. IRE 43, No. 10, 11711570.

Megaw, E. C. S. (1950), Scattering of electromagnetic waves by atmospheric turbulence, Nature 166, No. 4235, 1100-1104.

Saxton, J. A., J. A. Lane, R. W. Meadows, and P. A. Matthews (1964), Layer structure of the troposphere, Proc. Inst. Elec. Engrs. 111, No. 2, 275-283.

Smith, P. L., Jr. (1964), On the inference of atmospheric refractive index profiles from observations of radar angels, Proc. 1964 World Conf. Radio Meteorol. (Boulder, Colo.), 42-45.

Tatarski, V. I. (1961), Wave Propagation in a Turbulent Medium, ch. 4. (McGraw-Hill Book Co., Inc., New York, N.Y.).

Thompson, M. C., and M. J. Vetter (1958), Compact microwave refractometer for use in small aircraft, Rev. Sci. Instr. 29, No. 12, 1093-1096.

Wheelon, A. D. (1959), Radio-wave scattering by tropospheric irregularities, J. Res. NBS $63 D$ (Radio Prop.), No. 2, 205-233. 